\title{
DESIGN AND DEVELOPMENT OF GPS-GSM BASED Tracking SysteM WiTh GoOgLe MAP BASED MONITORING
}

\author{
Pankaj Verma ${ }^{1}$, J.S Bhatia ${ }^{2}$ \\ Centre for Development of Advanced Computing, Mohali, Punjab, India \\ pankajvermab35@yahoo.com \\ jsbhatia.cdac@gmail.com
}

\begin{abstract}
GPS is one of the technologies that are used in a huge number of applications today. One of the applications is tracking your vehicle and keeps regular monitoring on them. This tracking system can inform you the location and route travelled by vehicle, and that information can be observed from any other remote location. It also includes the web application that provides you exact location of target. This system enables us to track target in any weather conditions. This system uses GPS and GSM technologies. The paper includes the hardware part which comprises of GPS, GSM, Atmega microcontroller MAX 232, $16 \times 2$ LCD and software part is used for interfacing all the required modules and a web application is also developed at the client side. Main objective is to design a system that can be easily installed and to provide platform for further enhancement.
\end{abstract}

\section{KEYWORDS}

GPS, GSM, Tracking System

\section{INTRODUCTION}

In this urban life transportation is very common. A lot of mishappenings occur on the road every day .Therefore the need of security and monitoring is developed. To resolve such problems, a system is developed using GPS and GSM technologies and an application is introduced in this research work.

Various problems that we face:

1. In critical condition (when vehicle is stolen), one is confused what to do

2. If one has something expensive and he wants to check it regularly

3. To find the shortest path available

All these problems are overcome by the system.

This system has Global Positioning System (GPS) which will receive the coordinates from the satellites among other critical information. Tracking system is very important in modern world. This can be useful in soldier monitoring, tracking of the theft vehicle and various other applications. The system is microcontroller based that consists of a global positioning system (GPS) and global system for mobile communication (GSM). This project uses only one GPS device and a two way communication process is achieved using a GSM modem. GSM modem, provided with a SIM card uses the same communication process as we are using in regular phone.

DOI : $10.5121 /$ ijcsea.2013.3304 
The system is not limited to find the location of the target but also calculates the distance travelled b/w two stations.

This system is user friendly, easily installable, easily accessible and can be used for various other purposes. After installation system will locate target by the use of a Web application (HTML based application) in Google map. The system allows to track the target anytime and anywhere in any weather conditions.

\section{LITERATURE SURVEY}

Real-time tracking and management of vehicles has been a field of interest for many researchers and a lot of research work has been done for tracking system. Recently the various anti-theft modules like steering wheel locked equipment, network tracking system and traditional electronic alarm are developed along with client identification and real time performance monitoring.

The paper presented by El-Medany, W.; Al-Omary et al describes a real time tracking system that provides accurate localizations of the tracked vehicle with low cost. GM862 cellular quad band module is used for implementation. A monitoring server and a graphical user interface on a website is also developed using Microsoft SQL Server 2003 and ASP.net to view the proper location of a vehicle on a specific map. The paper also provides information regarding the vehicle status such as speed, mileage. ${ }^{[1]}$

Hu Jian-ming; Li Jie; Li Guang-Hui describes an automobile anti-theft system using GSM and GPS module. The system is developed using high speed mixed type single-chip C8051F120 and stolen automobile is detected by the use of vibration sensor. The system remains in contact with automobile owner through the GSM module, for the safety and reliability of automobile. ${ }^{[2]}$

Fleischer, P.B.; Nelson et al describes development and deployment of GPS (Global Positioning System)/GSM (Global System for Mobile Communications) based Vehicle Tracking and Alert System. This system allows inter-city transport companies to track their vehicles in real-time and provides security from armed robbery and accident occurrences. ${ }^{[4]}$

Le-Tien, T.; Vu Phung describes a system based on the Global Positioning System (GPS) and Global System for Mobile Communication (GSM). It describes the practical model for routing and tracking with mobile vehicle in a large area outdoor environment .The system includes the Compass sensor-YAS529 of Yamaha Company and Accelerator sensor-KXSC72050 of Koinix Company to acquire moving direction of a vehicle. The system will acquire positions of the vehicle via GPS receiver and then sends the data to supervised center by the SMS (Short Message Services) or GPRS (General Package Radio Service) service. The supervised center comprises of a development kit that supports GSM techniques-WMP100 of the Wavecom Company. Finally, the position of the mobile vehicle will be displayed on Google Map. ${ }^{[5]}$

\section{SYSTEM ARCHITECTURE}

It consists of two units one is transmitting side (vehicle unit) and other one is monitoring side.

\subsection{Description of transmitting unit:}

\subsubsection{GPS}

GPS modules are popularly used for navigation, positioning, time and other purposes. GPS antenna receives the location values from the satellites. GPS gives information about: 
1) Message transmission time

2) Position at that time

\subsubsection{GSM}

GSM modem is used for transmitting and receiving the data. SIM 300 is a tri- band GSM/GPRS engine. It works on various frequencies i.e. EGSM 900MHz, DCS $1800 \mathrm{MHz}$ and PCS $1900 \mathrm{MHz}$.

\subsubsection{Microcontroller}

The system uses a CMOS 8- bit microcontroller. It is based on RISC architecture. It comprises of $16 \mathrm{k}$ bytes of flash program memory, $1 \mathrm{~K}$ byte internal SRAM and 512 bytes EEPROM.

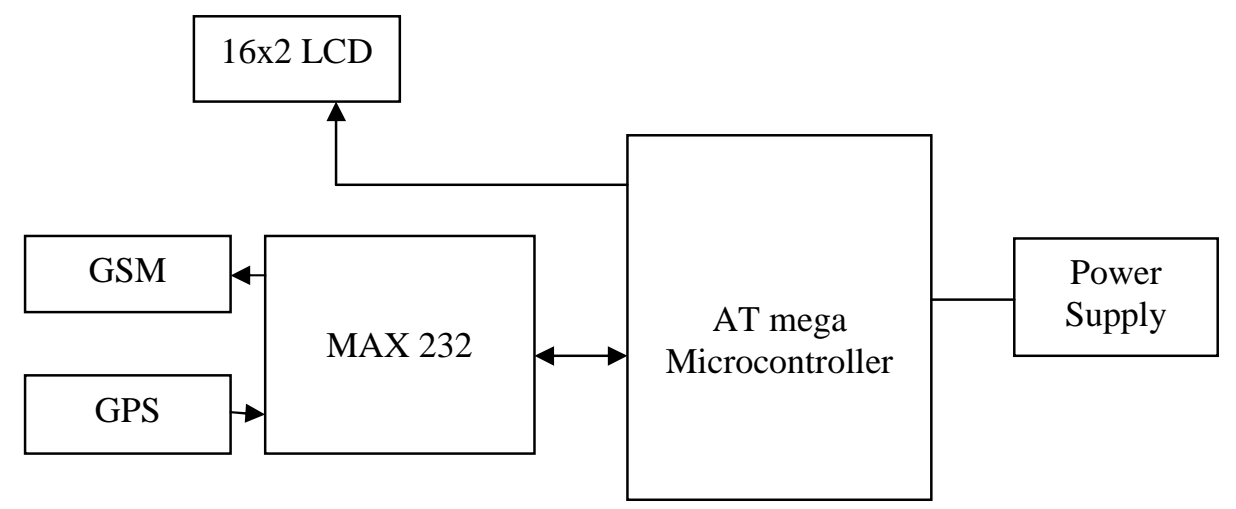

Figure 1.Architecture of transmitting unit

\subsubsection{MAX 232}

It is used for GSM, GPS and microcontroller to communicate serially

\subsubsection{6x2 LCD}

A $16 \times 2$ LCD is used for displaying location values. A $9 \mathrm{v}$ battery is used to power up the circuit.

\subsection{Monitoring unit}

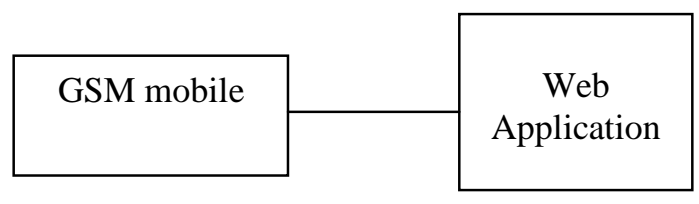

Figure 2.Monitoring unit Architecture

The monitoring unit consists of a GSM mobile and a Web Application. The GSM mobile will acquire the position of the vehicle (longitude and longitude) and then by typing those co-ordinates in web application owner of vehicle can get the exact location of the vehicle. The web application part is covered later in this paper. 


\section{HARDWARE DESIGN}

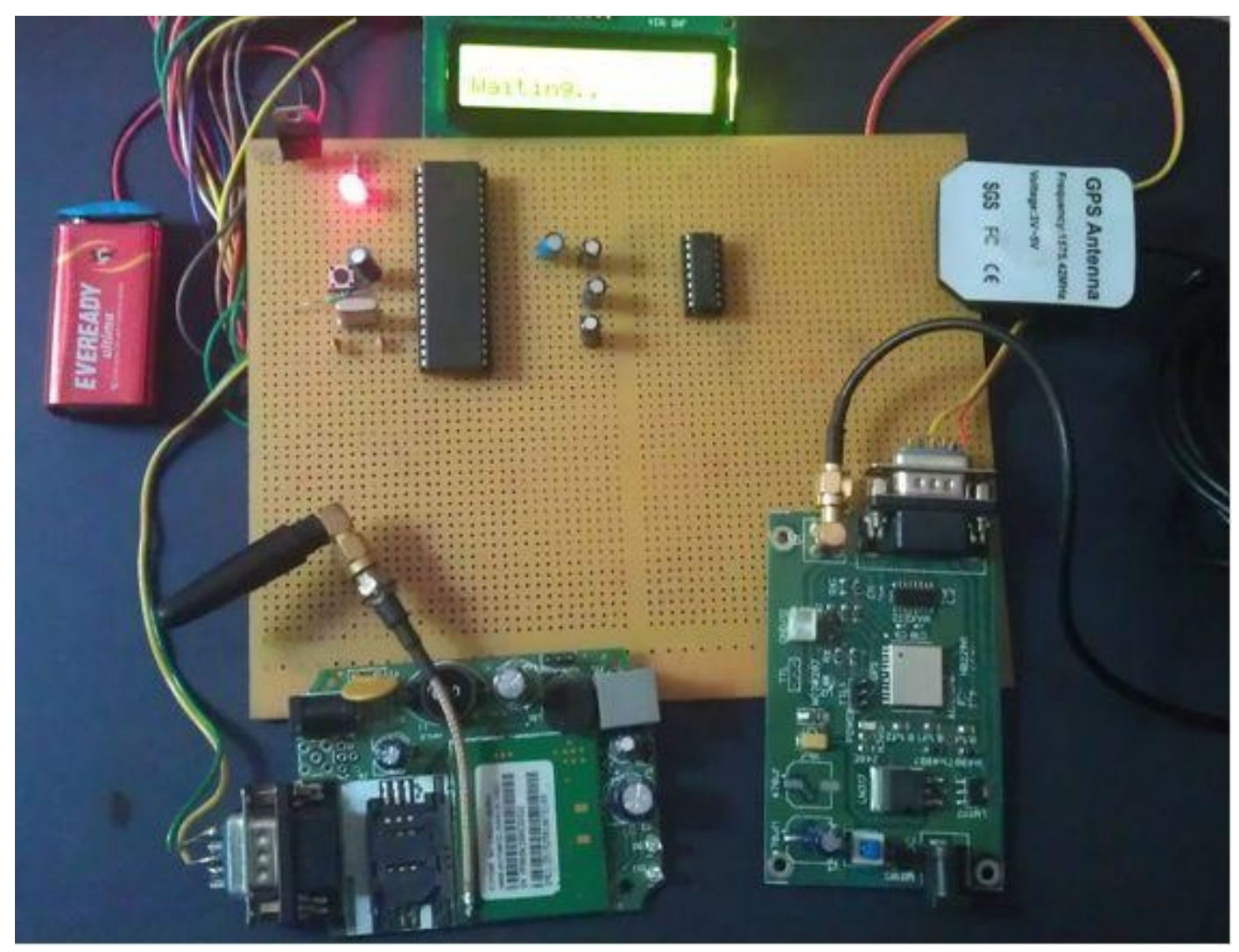

Figure 3.Hardware design

In this we are using a 40 pin ATmega16 microcontroller. It has four input-output ports. ATmega16 microcontroller is the heart of the project that is used for interfacing. Two pins are VCC pins and other two pins are at ground. Pin 9 is reset pin. A crystal oscillator of $12 \mathrm{MHz}$ is connected to the microcontroller. RS-232 protocol is used as serial communication between the microcontroller, GPS and GSM modem. A serial driver MAX232, 16 pin IC is used for converting RS-232 voltage levels into TTL voltage levels. There are four electrolytic capacitors which are used with MAX232. A 9V battery is used to power the circuit. A 7805 regulator is used to convert $9 \mathrm{~V}$ into $5 \mathrm{~V}$. The microcontroller and MAX232 are powered by $5 \mathrm{~V}$. LED indicates the presence of power supply.

\section{SoftWare Program}

The software programming is done in ' $\mathrm{C}$ 'language. Data (co-ordinates) received by GPS from the satellites is defined in the software. Decoding the NMEA (National Marine Electronics Association) protocol is the main purpose of developing this software. The mobile number of the user should be included in the software programming in order to receive the location values from the SIM card which we are using in GSM modem. The NMEA protocol consists of set of messages. These messages are ASCII character set. GPS receives data and present it in the form of ASCII comma - delimited message strings. '\$' sign is used at the starting of each message. The locations (latitude and longitude) have the format of ddmm.mmmm. i.e. .degrees minutes and decimal minutes. The software protocol consists of the GGA (global positioning system fixed 
data) and GLL (geographic position latitude/longitude). But in this system we are using CGA only. The flow chart of the system is given as:

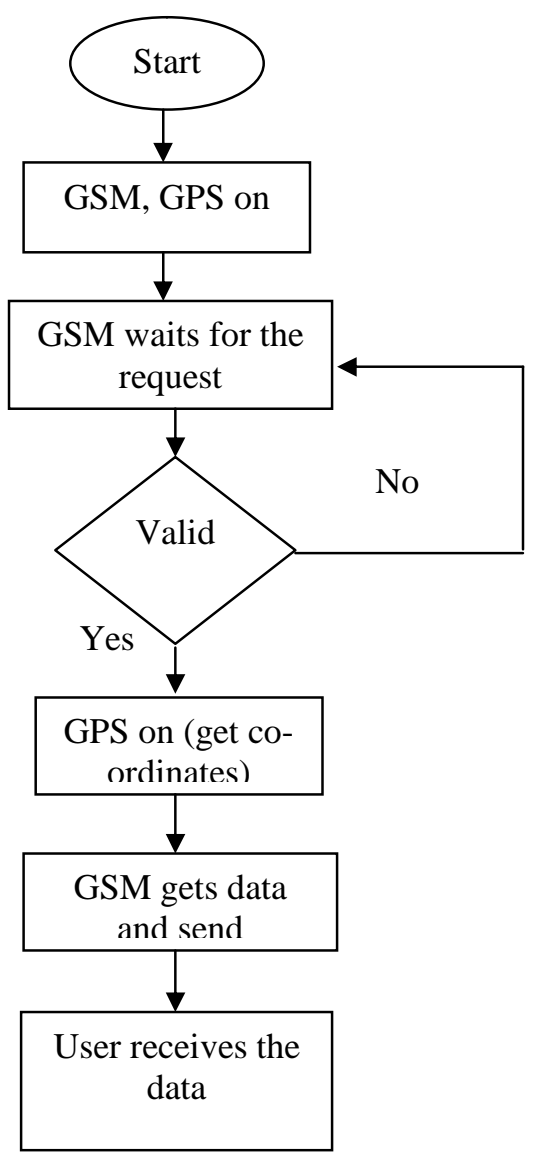

Figure 4.Program flow chart of the tracking system

\section{WeB APPLicAtion}

The web application named as 'Tracking System' is shown in figures that represents the complete output of the system. In this system two applications are developed that are linked to each other. First one is used to get the initial position of the vehicle (starting point) and as system will receive the different co-ordinates (longitude and latitude) switching to the next one will be done to get the distance travelled $\mathrm{b} / \mathrm{w}$ the two positions. The application will run on WAMP server and will run only if the internet is in use. WAMP server homepage is shown in diagram. 

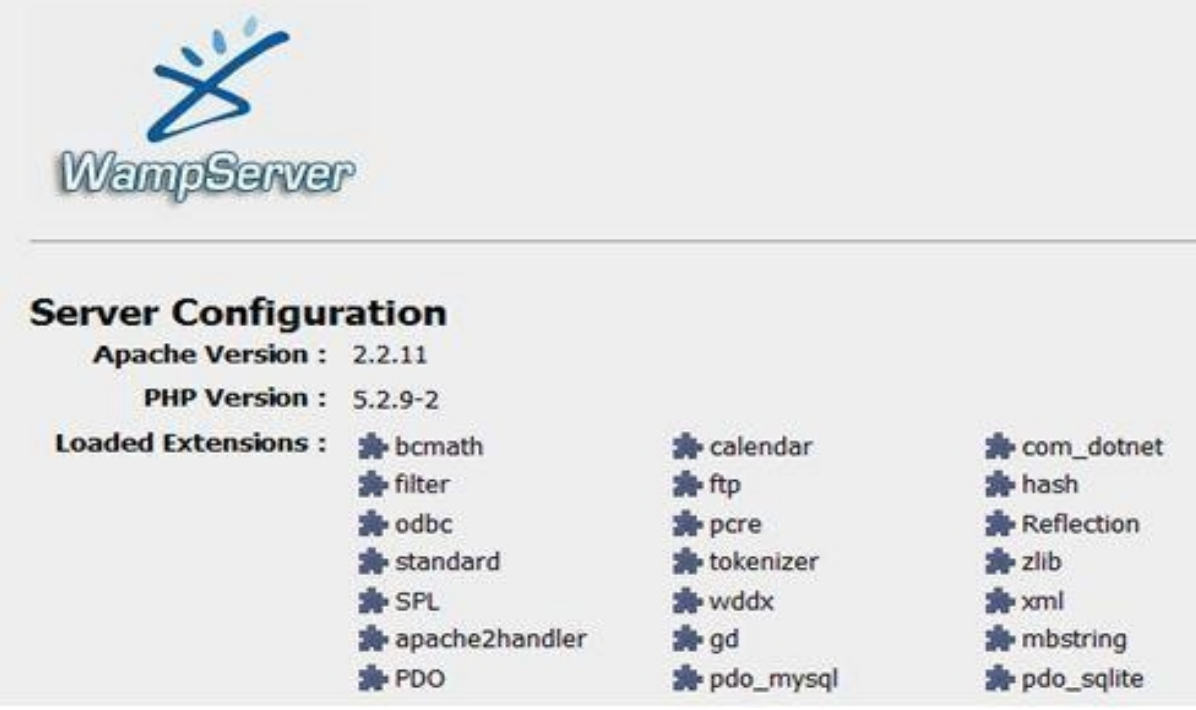

Figure 5.WAMP server home page

\subsection{Various features of web Application are:}

$>$ Both the applications are user friendly .i.e. new users can be easily comfortable.

$>$ Since the applications are HTML based user can easily modify according to his requirements

$>$ Gives the exact location of the target.

$>$ The applications also alerts about the distance travelled by the target and also the routes that are possible to reach to the target.

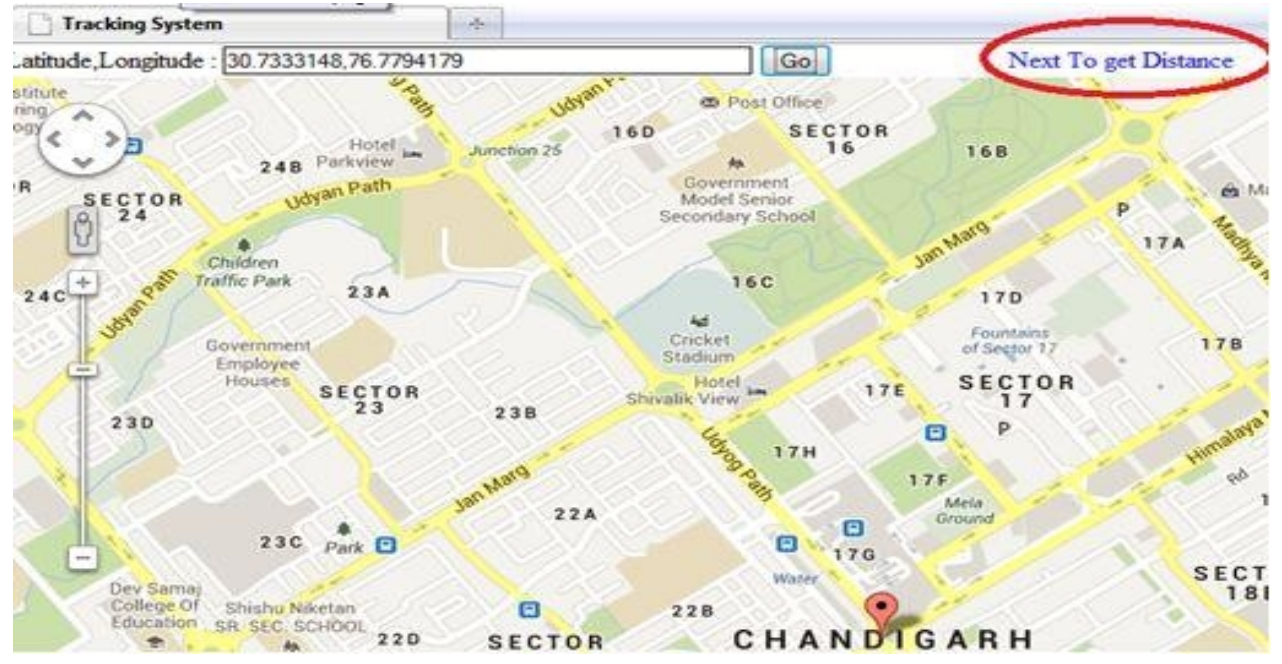

Figure 6.System Application 1

Figure 6 shows the first application where location will be found .As shown in figure by clicking on the 'next' button will open up next page, where the route and the distance travelled will be found. And by clicking on the 'Back' button will open up first one (page) as suggested in the fig. 7.Both the applications are HTML based. PHP is also used for some modifications. 


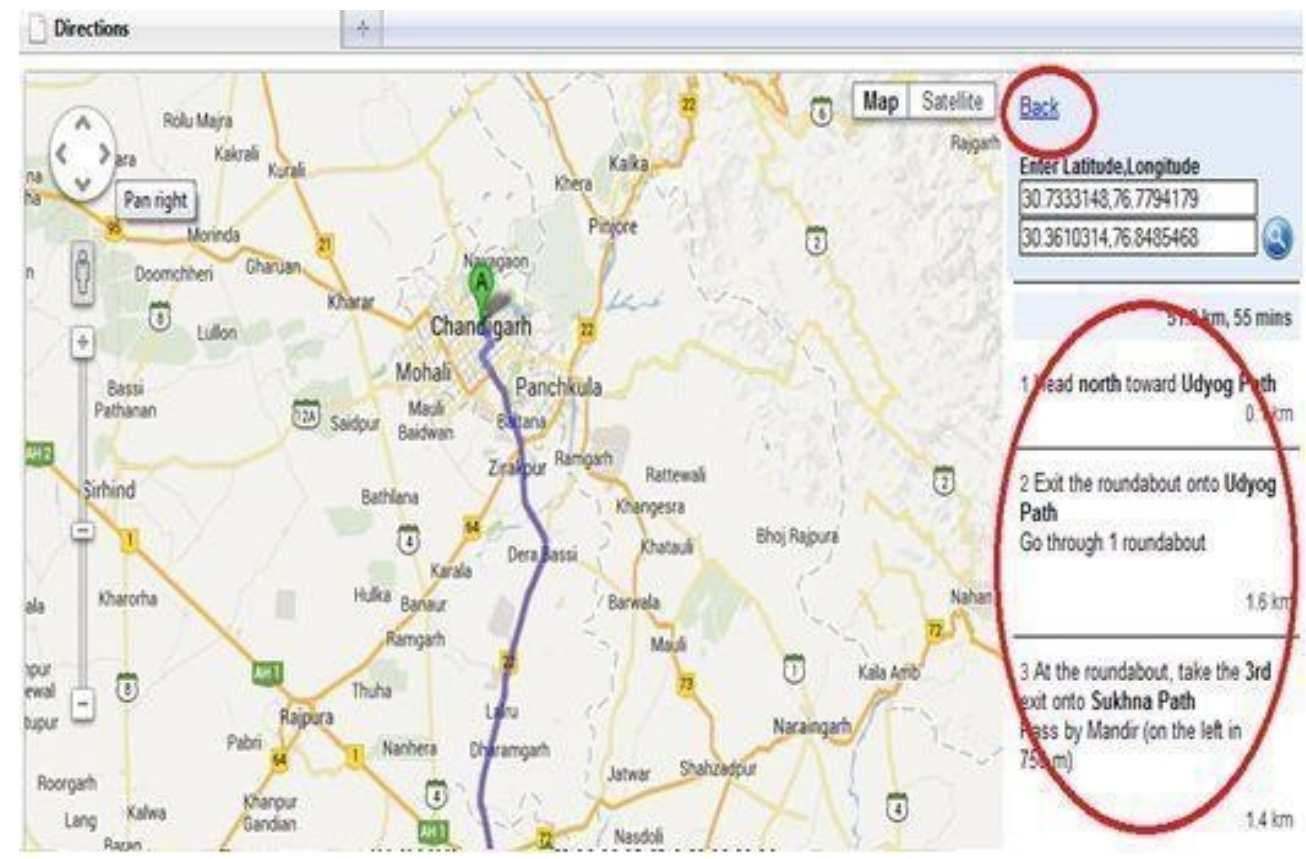

Figure 7.System Application 2

\section{CONCLUSION AND FUTURE SCOPE}

The project is all about controlling theft of a vehicle. The system is about making vehicle more secure by the use of GPS, GSM technology and a web application. The simulation is done by PROTEUS software. It can also be beneficial for:

1. Parents to look after their children.

2. To track animals in jungles

3. Delivery services

4. Cops department and fire services

This project can be further enhanced by the use of camera and by developing a mobile based application to get the real time view of the vehicle instead to check it on PC, which would be more convenient for the user to track the target.

\section{ACKNOWLEDGEMENT}

We would like to extend a special thank to C-DAC Mohali for providing us means to carry out our research work in precious way. We are also grateful to MHRD, Govt. of India for providing us a platform to do our research work.

\section{REFERENCES}

[1] El-Medany,W.;Al-Omary,A.;Al-Hakim,R.;Al-Irhayim,S.;Nusaif,M.,"A Cost Effective Real-Time Tracking System Prototype Using Integrated GPS/GPRS Module," Wireless and Mobile Communications (ICWMC), 2010 6th International Conference on,vol.,no.,pp.521,525,20-25 Sept.2010 
[2] Hu Jian-ming; Li Jie; Li Guang-Hui, "Automobile Anti-theft System Based on GSM and GPS Module," Intelligent Networks and Intelligent Systems (ICINIS), 2012 Fifth International Conference on , vol., no., pp.199,201, 1-3 Nov. 2012

[3] Nagaraja, B. G.; Rayappa, R.; Mahesh, M.; Patil, C.M.; Manjunath, T. C., "Design \& Development of a GSM Based Vehicle Theft Control System," Advanced Computer Control, 2009. ICACC '09. International Conference on , vol., no., pp.148,152, 22-24 Jan. 2009

[4] Fleischer, P.B.; Nelson, A.Y.; Sowah, R.A.; Bremang, A., "Design and development of GPS/GSM based vehicle tracking and alert system for commercial inter-city buses," Adaptive Science \& Technology (ICAST), 2012 IEEE 4th International Conference on , vol., no., pp.1,6, 25-27 Oct. 2012

[5] Le-Tien, T.; Vu Phung-The, "Routing and Tracking System for Mobile Vehicles in Large Area," Electronic Design, Test and Application, 2010. DELTA '10. Fifth IEEE International Symposium on , vol., no., pp.297,300, 13-15 Jan. 2010

[6] Iman M. Almomani, Nour Y. Alkhalil, Enas M. Ahmad, Rania M. Jodeh "Ubiquitous GPS Vehicle Tracking and Management System", IEEE Jordan Conference on Applied Electrical Engineering and Computing Technologies (AEECT) 2011

[7] Abed khan M.E.(Student), , Ravi Mishra, "GPS - GSM Based Tracking System" SSCET, CSVTU, Bhilai, India International Journal of Engineering Trends and Technology- vol.3,no.,pp,161-164, 2012

[8] El-Medany, W.M.; Alomary, A.; Al-Hakim, R.; Al-Irhayim, S.; Nousif, M., "Implementation of GPRSBased Positioning System Using PIC Microcontroller," Computational Intelligence, Communication Systems and Networks (CICSyN), 2010 Second International Conference on , vol., no., pp.365,368, 28-30 July 2010

[9] Iman M. Almomani, Nour Y. Alkhalil, Enas M. Ahmad, Rania M. Jodeh "Ubiquitous GPS Vehicle Tracking and Management System", IEEE Jordan Conference on Applied Electrical Engineering and Computing Technologies (AEECT) 2011

[10] Lita, I.; Cioc, I.B.; Visan, D.A., "A New Approach of Automobile Localization System Using GPS and GSM/GPRS Transmission," Electronics Technology, 2006. ISSE '06. 29th International Spring Seminar on , vol., no., pp.115,119, 10-14 May 2006

[11] Parvez, M.Z.; Ahmed, K.Z.; Mahfuz, Q.R.; Rahman, M.S., "A theoretical model of GSM network based vehicle tracking system," Electrical and Computer Engineering (ICECE), 2010 International Conference on , vol., no., pp.594,597, 18-20 Dec. 2010

[12] Sadagopan, V.K.; Rajendran, U.; Francis, A.J., "Anti theft control system design using embedded system," Vehicular Electronics and Safety (ICVES), 2011 IEEE International Conference on, vol., no., pp.1, 5, 10-12 July 2011

[13] www.htmlgoodies.com

[14] http://maps.google.com/

[15] GSMWorld.[Online].Retrieved 15/7/2010 available:http://www.gsmworld.com/technology/faq.shtml

[16] SIM 300 data sheet, SIM 300-HD_V1.06

[17] Datasheet of Atmega 16 microcontroller, Rev. 2467X-AVR-06/11

[18] MAX 232 datasheet, February 1989- revised 2012

\section{AUTHORS}

Pankaj Verma is pursuing M.Tech. in Electronic Product Design \& Technology from Centre for Development of Advanced Computing (C-DAC) Mohali. His area of research is in communication system.

Mr. J.S. Bhatia is working as Executive Director at C-DAC Mohali. He focuses on cutting edge solutions in various domains like health informatics, multimedia technology and professional electronics.

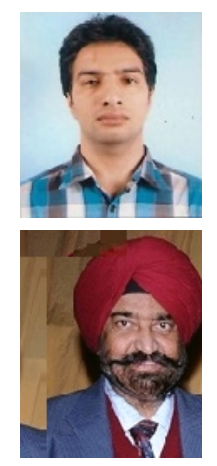

